\title{
Lidstone-Euler Second-Type Boundary Value Problems: Theoretical and Computational Tools
}

\author{
Francesco Aldo Costabile, Maria Italia Gualtieri and Anna Napoli
}

Dedicated to Professor Francesco Altomare on his 70th birthday.

\begin{abstract}
General nonlinear high odd-order differential equations with Lidstone-Euler boundary conditions of second type are treated both theoretically and computationally. First, the associated interpolation problem is considered. Then, a theorem of existence and uniqueness of the solution to the Lidstone-Euler second-type boundary value problem is given. Finally, for a numerical solution, two different approaches are illustrated and some numerical examples are included to demonstrate the validity and applicability of the proposed algorithms.
\end{abstract}

Mathematics Subject Classification. 65L10, 11B68, 11 B83.

Keywords. Boundary value problem, Lidstone polynomials, Euler polynomials, Bernstein polynomials, interpolation, extrapolation.

\section{Introduction}

Boundary value problems (BVPs) with higher order differential equations play an important role in a variety of different branches of applied mathematics, engineering, and many other fields of advanced physical sciences. For examples, third-order BVPs arise in several physical problems, such as the deflection of a curved beam, the motion of rockets, thin-film flows, electromagnetic waves, or gravity-driven flows (see $[3,4]$ and references therein). Fifth-order differential equations are used in mathematical modelling of viscoelastic flows [17]. Seventh-order BVPs arise in modelling induction motor with two rotor circuits [26]. Nineth-order BVPs are known to arise in hydrodynamic, hydromagnetic stability, and mathematical modelling of AFTI-F16 fighters $[2,23]$. For more details on accuracy of high-order BVPs, see $[6,13-$ $15,19,20,22,33,34$, and references therein]. 
In this paper, we will consider the boundary value problem

$$
\left\{\begin{array}{l}
y^{(2 r+1)}(x)=f\left(x, y(x), y^{\prime}(x), \ldots, y^{(q)}(x)\right) \\
y^{(2 i+1)}(0)=\alpha_{i}, \quad i=0, \ldots, r-1 \\
y^{(2 i)}(1)=\beta_{i}, \quad i=0, \ldots, r
\end{array}\right.
$$

with $0 \leq q \leq 2 r$ fixed; $\alpha_{i}, i=0, \ldots, r-1$, and $\beta_{i}, i=0, \ldots, r$, finite real constants. The function $f$ is defined and continuous in $[0,1] \times D, D \subset \mathbb{R}^{q+1}$.

The existence and uniqueness of solution of high-order BVPs are discussed in [1], but there numerical methods and examples are only mentioned. Problem (1.1) does not appear in [1], for the particularity of the boundary conditions. The boundary conditions in (1.1) have physical meanings. For example, for $r=1$, they represent the position and acceleration at the end point and the velocity at the starting point of the system. However, to the best of authors' knowledge, there are no concret physical problems at least in the mathematical literature.

We call the BVP (1.1) Lidstone-Euler second-type boundary value problem in contrast to Lidstone-Euler (first-type) boundary value problem in [10].

The interpolatory theory and qualitative as well as quantitative study of BVPs are directly connected $[1,7,10,16]$. The boundary conditions in (1.1), that is

$$
y^{(2 i+1)}(0)=\alpha_{i}, \quad i=0, \ldots, r-1, \quad y^{(2 i)}(1)=\beta_{i}, \quad i=0, \ldots, r,
$$

represent the Birkhoff-type interpolatory problem with the following incidence matrix, in Schoenberg notation: [28]:

$$
\left(\begin{array}{lllll}
0 & 1 & 0 & 1 & \cdots \\
1 & 0 & 1 & 0 & \cdots
\end{array}\right)
$$

The corresponding interpolation series has been considered in $[24,25$, $27,28]$, and in [36] for analogous problems.

Our study on the BVP (1.1) starts from the interpolatory problem (1.2).

The paper is organized as follows: in Sect. 2, we consider the LidstoneEuler interpolation problem (1.2) [8,11] using the Lidstone-Euler secondtype (or even) polynomials, and we give some new results concerning the bounds of error and the convergence. In Sect. 3, we consider the existence and uniqueness of the solution of problem (1.1). In Sect. 4, we discuss some computational aspects and we give two algorithms for computing a numerical solution of the problem. In Sect. 5, we present some numerical examples to illustrate the applicability of the proposed methods. The results clearly show that the described procedures are able to produce good results in terms of accuracy. Finally, some conclusions are given. 


\section{Lidstone-Euler Second-Type Polynomials and Related Interpolation Problem}

Lidstone-Euler second-type polynomials $\mathscr{S}_{k}(x)$, also called Lidstone-Euler even polynomials, have been introduced in $[8,11,12]$. They satisfy the BVP of second order

$$
\left\{\begin{array}{l}
\mathscr{S}_{k}^{\prime \prime}(x)=2 k(2 k-1) \mathscr{S}_{k-1}(x), \quad k \geq 1, \\
\mathscr{S}_{k}(1)=0, \quad k \geq 1, \quad \mathscr{S}_{k}^{\prime}(0)=0, \quad k \geq 0 .
\end{array}\right.
$$

Polynomials $\mathscr{S}_{k}(x)$ are connected to Euler polynomials by the identity

$$
\mathscr{S}_{k}(x)=2^{2 k} E_{2 k}\left(\frac{x+1}{2}\right), \quad k=0,1, \ldots,
$$

where $E_{k}(x)$ is the classic Euler polynomial of degree $k$ [8]. Moreover, $\mathscr{S}_{k}(x)$ satisfies

$$
\mathscr{S}_{k}(x)=\frac{\varepsilon_{k}^{\prime}(x)}{2 k+1}
$$

$\varepsilon_{k}(x)$ being the Lidstone-Euler first-type polynomial $[8,10,11]$.

The first polynomials $\mathscr{S}_{k}(x)$ are

$$
\begin{aligned}
& \mathscr{S}_{0}(x)=1, \\
& \mathscr{S}_{1}(x)=-1+x^{2}, \\
& \mathscr{S}_{2}(x)=5-6 x^{2}+x^{4}, \\
& \mathscr{S}_{3}(x)=-61+75 x^{2}-15 x^{4}+x^{6}, \\
& \mathscr{S}_{4}(x)=1385-1708 x^{2}+350 x^{4}-28 x^{6}+x^{8} .
\end{aligned}
$$

Relations (2.1) and (2.2) justify the name Lidstone-Euler type given to the sequence $\left\{\mathscr{S}_{k}\right\}_{k}$. This sequence is important, because it acts as a fundamental polynomial sequence for the Birkhoff interpolation problem given by the incidence matrix (1.3).

Theorem 1. For the Lidstone-Euler second-type polynomials, the following identity holds:

$$
\mathscr{S}_{k}(x)=(2 k) ! \int_{0}^{1} g_{k}(x, t) \mathrm{d} t, \quad k \geq 1,
$$

where

$$
\begin{aligned}
g_{1}(x, t) & = \begin{cases}x-1 & t \leq x \\
t-1 & t>x,\end{cases} \\
g_{k}(x, t) & =\int_{0}^{1} g_{1}(x, s) g_{k-1}(s, t) \mathrm{d} s, \quad k \geq 2 .
\end{aligned}
$$

Proof. The proof follows by induction. For $k=1$, the thesis is trivial. For $k \geq$ 2 , we observe that the polynomial $\mathscr{S}_{k+1}(x)$ is the solution of the boundary value problem

$$
\left\{\begin{array}{l}
\mathscr{S}_{k+1}^{\prime \prime}(x)=(2 k+2)(2 k+1) \mathscr{S}_{k}(x) \\
\mathscr{S}_{k+1}(1)=\mathscr{S}_{k+1}^{\prime}(0)=0
\end{array}\right.
$$


From the inductive hypothesis, we have

$$
\left\{\begin{array}{l}
\mathscr{S}_{k+1}^{\prime \prime}(x)=(2 k+2) ! \int_{0}^{1} g_{k}(x, t) \mathrm{d} t \\
\mathscr{S}_{k+1}(1)=\mathscr{S}_{k+1}^{\prime}(0)=0 .
\end{array}\right.
$$

From the theory of differential equations, the solution of (2.7) is

$$
\begin{aligned}
\mathscr{S}_{k+1}(x) & =\int_{0}^{1} g_{1}(x, s)\left[(2 k+2) ! \int_{0}^{1} g_{k}(s, t) \mathrm{d} t\right] \mathrm{d} s \\
& =(2 k+2) ! \int_{0}^{1}\left[\int_{0}^{1} g_{1}(x, s) g_{k}(s, t) \mathrm{d} s\right] \mathrm{d} t \\
& =(2 k+2) ! \int_{0}^{1} g_{k+1}(x, t) \mathrm{d} t .
\end{aligned}
$$

Thus, the thesis follows.

Remark 1. From (2.4), $g_{1}(x, t) \leq 0,0 \leq x, t \leq 1$. Thus, from (2.5),

$$
0 \leq(-1)^{k} g_{k}(x, t)=\left|g_{k}(x, t)\right|, \quad 0 \leq x, t \leq 1 .
$$

Hence, in view of $(2.3)$, we get $(-1)^{k} \mathscr{S}_{k}(x) \geq 0,0 \leq x \leq 1$.

Theorem 2. The Lidstone-Euler second-type polynomials can be written as

$$
\mathscr{S}_{k}(x)=(2 k) ! \int_{0}^{1} t G_{k-1}(x, t) \mathrm{d} t, \quad k \geq 1,
$$

where

$$
\begin{aligned}
& G_{0}(x, t)= \begin{cases}0 & t \leq x \\
-1 & t>x,\end{cases} \\
& G_{k}(x, t)=\int_{0}^{1} g_{1}(x, s) G_{k-1}(s, t) \mathrm{d} s, \quad k \geq 1,
\end{aligned}
$$

with $g_{1}$ defined as in (2.4).

Proof. Relation (2.8) can be proved by induction. For $k=1$, it is trivially true. For $k>1$, from (2.8) and the inductive hypothesis, the boundary value problem (2.6) can be written as

$$
\left\{\begin{array}{l}
\mathscr{S}_{k+1}^{\prime \prime}(x)=(2 k+2) ! \int_{0}^{1} t G_{k-1}(x, t) \mathrm{d} t \\
\mathscr{S}_{k+1}(1)=\mathscr{S}_{k+1}^{\prime}(0)=0 .
\end{array}\right.
$$

The solution of (2.11) is

$$
\begin{aligned}
\mathscr{S}_{k+1}(x) & =\int_{0}^{1} g_{1}(x, s)\left[(2 k+2) ! \int_{0}^{1} t G_{k-1}(s, t) \mathrm{d} t\right] \mathrm{d} s \\
& =(2 k+2) ! \int_{0}^{1}\left[\int_{0}^{1} g_{1}(x, s) G_{k-1}(s, t) \mathrm{d} s\right] t \mathrm{~d} t \\
& =(2 k+2) ! \int_{0}^{1} t G_{k}(x, t) \mathrm{d} t
\end{aligned}
$$

and this completes the proof. 
Remark 2. Since $g_{1}(x, t) \leq 0$, from $(2.10)$ it holds $(-1)^{k+1} G_{k}(x, t) \geq 0$.

Proposition 1. Let $G_{k}(x, t)$ be the function defined in (2.9)-(2.10). Then

(i) $G_{k}(1, t)=0, \quad k \geq 1$;

(ii) $\left.\frac{\partial}{\partial x} G_{k}(x, t)\right|_{x=0}=0, \quad k \geq 0$;

(iii) $\frac{\partial^{2 s}}{\partial x^{2 s}} G_{k}(x, t)=G_{k-s}(x, t), \quad s=0, \ldots, k-1, \quad k \geq 1$;

(iv) $\frac{\partial^{2 s+1}}{\partial x^{2 s+1}} G_{k}(x, t)=\frac{\partial}{\partial x} G_{k-s}(x, t), \quad s=0, \ldots, k-1, \quad k \geq 1$.

Proof. The first two identities follow from the definition of $G_{k}(x, t)$ and the boundary conditions in (2.1). Property $(i i i)$ is obtained from the first of $(2.1)$ and Theorem 2. Relation (iv) follows from (iii).

Proposition 2. For the function $G_{k}(x, t)$, the following inequalities hold:

$$
\begin{aligned}
& \int_{0}^{1}\left|G_{k}(x, t)\right| \mathrm{d} t \leq \frac{1}{2^{k}}, \quad k \geq 0 \\
& \int_{0}^{1}\left|\frac{\partial}{\partial x} G_{k}(x, t)\right| \mathrm{d} t \leq \frac{1}{2^{k-1}}, \quad k \geq 1 .
\end{aligned}
$$

Proof. The proof of (2.12) follows by induction. For $k=0$, the thesis is trivial. For $k \geq 1$, from (2.4), (2.10), and the inductive hypothesis, we get

$$
\begin{aligned}
\int_{0}^{1}\left|G_{k+1}(x, t)\right| \mathrm{d} t & =\int_{0}^{1}\left|\int_{0}^{1} g_{1}(x, s) G_{k}(s, t) \mathrm{d} s\right| \mathrm{d} t \\
& \leq \int_{0}^{1}\left|g_{1}(x, s)\right|\left(\int_{0}^{1}\left|G_{k}(s, t)\right| \mathrm{d} t\right) \mathrm{d} s \\
& \leq \frac{1}{2^{k}} \int_{0}^{1}\left|g_{1}(x, s)\right| \mathrm{d} s \leq \frac{1}{2^{k+1}}
\end{aligned}
$$

To prove property (2.13), observe that

$$
\begin{aligned}
G_{k}(x, t) & =\int_{0}^{1} g_{1}(x, s) G_{k-1}(s, t) \mathrm{d} s \\
& =\int_{0}^{x}(x-1) G_{k-1}(s, t) \mathrm{d} s+\int_{x}^{1}(s-1) G_{k-1}(s, t) \mathrm{d} s .
\end{aligned}
$$

By differentiating, we obtain

$$
\frac{\partial}{\partial x} G_{k}(x, t)=\int_{0}^{x} G_{k-1}(s, t) \mathrm{d} s .
$$

Hence

$$
\int_{0}^{1}\left|\frac{\partial}{\partial x} G_{k}(x, t)\right| \mathrm{d} t \leq \int_{0}^{1}\left(\int_{0}^{x}\left|G_{k-1}(s, t)\right| \mathrm{d} s\right) \mathrm{d} t=\int_{0}^{x}\left(\int_{0}^{1}\left|G_{k-1}(s, t)\right| \mathrm{d} t\right) \mathrm{d} s .
$$

From (2.12), it follows:

$$
\int_{0}^{1}\left|\frac{\partial}{\partial x} G_{k}(x, t)\right| \mathrm{d} t \leq \frac{1}{2^{k-1}} \int_{0}^{1} \mathrm{~d} t \leq \frac{1}{2^{k-1}} .
$$

The following two results have been proved in [11]. 
Theorem 3. The polynomial $Q_{\ell}$ defined by

$$
Q_{\ell}(x)=\sum_{i=0}^{\ell} \frac{\tilde{\beta}_{i}}{(2 i) !} \mathscr{S}_{i}(x)+\sum_{i=0}^{\ell-1} \frac{\tilde{\alpha}_{i}}{(2 i+2) !} \mathscr{S}_{i+1}^{\prime}(1-x)
$$

is the unique polynomial of degree $2 \ell$ that satisfies the interpolatory conditions

$$
Q_{\ell}^{(2 i+1)}(0)=\tilde{\alpha}_{i}, \quad i=0, \ldots, \ell-1, \quad Q_{\ell}^{(2 i)}(1)=\tilde{\beta}_{i}, \quad i=0, \ldots, \ell,
$$

with $\tilde{\alpha}_{i}, i=0, \ldots, \ell-1$ and $\tilde{\beta}_{i}, i=0, \ldots, \ell$, given real numbers.

Hence, we call $Q_{\ell}(x)$ the Lidstone-Euler type interpolant polynomial of second kind and the conditions (2.15) the Lidstone-Euler type interpolation conditions of second kind.

Corollary 1. Let $f$ be a $2 \ell$-differentiable function in $[0,1]$. The polynomial

$$
Q_{\ell}[f](x)=\sum_{i=0}^{\ell} \frac{f^{(2 i)}(1)}{(2 i) !} \mathscr{S}_{i}(x)+\sum_{i=0}^{\ell-1} \frac{f^{(2 i+1)}(0)}{(2 i+2) !} \mathscr{S}_{i+1}^{\prime}(1-x)
$$

is the unique polynomial of degree $2 \ell$, such that

$$
Q_{r}^{(2 i+1)}[f](0)=f^{(2 i+1)}(0), \quad i=0, \ldots, \ell-1, \quad Q_{r}^{(2 i)}[f](1)=f^{(2 i)}(1), \quad i=0, \ldots, \ell .
$$

The polynomial $Q_{\ell}[f](x)$ is called the Lidstone-Euler interpolant polynomial of second kind for the function $f$.

Proposition 3. For the derivatives of the Lidstone-Euler type interpolant polynomial of second kind, there exist constants $C_{2 s}$ and $C_{2 s+1}$, such that $\left|Q_{\ell}^{(2 s)}[f](x)\right| \leq C_{2 s}, \quad\left|Q_{\ell}^{(2 s+1)}[f](x)\right| \leq C_{2 s+1}, \quad 0 \leq x \leq 1, \quad s=0, \ldots, \ell-1$, with

$$
C_{2 s}=\frac{\pi^{2 s+2}}{3 \cdot 2^{2 s}}\left[\sum_{i=2 s-2}^{\ell} \frac{\left|f^{(2 i)}(1)\right|}{(2 i-2 s) !}\left(\frac{2}{\pi}\right)^{2 i+1}+\sum_{i=2 s-2}^{\ell-1} \frac{\left|f^{(2 i+1)}(0)\right|}{(2 i-2 s+1) !}\left(\frac{2}{\pi}\right)^{2 i+2}\right]
$$

and

$$
C_{2 s+1}=\frac{\pi^{2 s}}{3 \cdot 2^{2 s}}\left[\sum_{i=2 s-2}^{\ell} \frac{\left|f^{(2 i)}(1)\right|}{(2 i-2 s-1) !}\left(\frac{2}{\pi}\right)^{2 i}+4 \sum_{i=2 s-2}^{\ell-1} \frac{\left|f^{(2 i+1)}(0)\right|}{(2 i-2 s) !}\left(\frac{2}{\pi}\right)^{2 i-1}\right] .
$$

Proof. The proof follows after easy calculations tacking into account the inequality $\left|E_{n}(x)\right| \leq \frac{2}{3 \pi^{n-1}}[21$, p. 303].

For any $x \in[0,1]$, we can define the remainder as

$$
T_{\ell}[f](x)=f(x)-Q_{\ell}[f](x), \quad r \geq 1 .
$$


Theorem 4. If $f \in C^{2 \ell+1}[0,1]$, the following identity holds:

$$
T_{\ell}[f](x)=\int_{0}^{1} f^{(2 \ell+1)}(t) G_{\ell}(x, t) \mathrm{d} t
$$

with $G_{\ell}(x, t)$ defined in $(2.10)$.

Proof. From definition, $T_{\ell}^{(2 \ell+1)}[f](x)=f^{(2 \ell+1)}(x)$. By integrating in $[x, 1]$, we get $T_{\ell}^{(2 \ell)}[f](x)=f^{(2 \ell)}(x)-f^{(2 \ell)}(1)=-\int_{x}^{1} f^{(2 \ell+1)}(t) \mathrm{d} t=\int_{0}^{1} G_{0}(x, t) f^{(2 \ell+1)}(t) \mathrm{d} t$, with $G_{0}(x, t)$ defined as in $(2.9)$.

For the second derivative of $T_{\ell}^{(2 \ell-2)}[f](x)$, we have

$$
\left(T_{\ell}^{(2 \ell-2)}\right)^{\prime \prime}[f](x)=T_{\ell}^{(2 \ell)}[f](x)=\int_{0}^{1} G_{0}(x, t) f^{(2 \ell+1)}(t) \mathrm{d} t .
$$

Moreover, from (2.17) and (2.20),

$$
T_{\ell}^{(2 \ell-2)}[f](1)=\left[\left(T_{\ell}^{(2 \ell-2)}\right)^{\prime}[f](x)\right]_{x=0}=0 ;
$$

hence

$$
\begin{aligned}
T_{\ell}^{(2 \ell-2)} & {[f](x)=\int_{0}^{1} g_{1}(x, s) \int_{0}^{1} G_{0}(s, t) f^{(2 \ell+1)}(t) \mathrm{d} t \mathrm{~d} s } \\
= & \int_{0}^{1}\left(\int_{0}^{1} g_{1}(x, s) G_{0}(s, t) \mathrm{d} s\right) f^{(2 \ell+1)}(t) \mathrm{d} t=\int_{0}^{1} G_{1}(x, t) f^{(2 \ell+1)}(t) \mathrm{d} t .
\end{aligned}
$$

By repeating $s$ times the same procedure, we get (2.21).

Theorem 5. (Cauchy representation) If $f \in C^{2 \ell+1}[0,1]$, there exists $\xi \in$ $(0,1)$, such that

$$
T_{\ell}[f](x)=f^{(2 \ell+1)}(\xi) \int_{0}^{1} G_{\ell}(x, t) \mathrm{d} t .
$$

Proof. The result follows from the mean value theorem for integrals, since $f \in C^{2 \ell+1}[0,1]$, and Remark 2 .

We can derive a different representation of the remainder.

Theorem 6. (Peano's representation of the remainder) [11] If $f \in C^{2 \ell+1}[0,1]$, the following identity holds

$$
T_{\ell}[f](x)=\int_{0}^{1} K_{\ell}(x, t) f^{(2 \ell+1)}(t) \mathrm{d} t,
$$

where

$$
K_{\ell}(x, t)=\frac{1}{(2 \ell) !}\left[(x-t)_{+}^{2 \ell}-Q_{\ell}\left[(x-t)_{+}^{2 \ell}\right](x)\right]
$$

$(\cdot)_{+}$being the known truncated power function [18]. 
Corollary 2. [11] For the Peano kernel $K_{\ell}(x, t)$, we get

$$
K_{\ell}(x, t)=\frac{(x-t)_{+}^{2 \ell}}{(2 \ell) !}-\sum_{i=0}^{\ell} \frac{(1-t)^{2(\ell-i)}}{(2(\ell-i)) !(2 i) !} \mathscr{S}_{i}(x), \quad r \geq 1 ;
$$

that is

$$
K_{\ell}(x, t)= \begin{cases}-\sum_{i=0}^{\ell-1} \frac{t^{2(\ell-i)-1}}{(2(\ell-i)-1) !(2 i+2) !} \mathscr{S}_{i+1}^{\prime}(1-x), & t \leq x \\ -\sum_{i=0}^{\ell} \frac{(1-t)^{2(\ell-i)}}{(2(\ell-i)) !(2 i) !} \mathscr{S}_{i}(x), & t>x .\end{cases}
$$

Proposition 4. For any $\ell \geq 1$, we get

$$
G_{\ell}(x, t)=K_{\ell}(x, t) .
$$

Proof. The thesis follows from (2.21), (2.22) and the uniqueness of Peano's kernel.

If we set

$$
M_{\ell}=\max _{0 \leq t \leq 1}\left|f^{(2 \ell+1)}(t)\right|,
$$

the following theorems provide bounds for the remainder and its derivatives.

Theorem 7. With the previous hypotheses and notations, the following bound holds:

$$
\left|T_{\ell}[f](x)\right| \leq M_{\ell} \int_{0}^{1}\left|G_{\ell}(x, t)\right| \mathrm{d} t \leq \frac{1}{2^{\ell}} M_{\ell} .
$$

Proof. The thesis follows from Cauchy representation and Proposition 2.

Theorem 8. With the previous hypothesys and notations, the following bounds hold:

$$
\begin{aligned}
& \left|T_{\ell}^{(2 i)}[f](x)\right| \leq \gamma_{\ell, 2 i} M_{\ell}, \quad i=0, \ldots, \ell, \\
& \left|T_{\ell}^{(2 i+1)}[f](x)\right| \leq \gamma_{\ell, 2 i+1} M_{\ell}, \quad i=0, \ldots, \ell-1,
\end{aligned}
$$

where $\gamma_{\ell, 2 i}=\frac{1}{2^{\ell-i}}, \gamma_{\ell, 2 i+1}=\frac{1}{2^{\ell-i+1}}$. It also holds

$$
\left|T_{\ell}^{(k)}[f](x)\right| \leq M_{\ell} \gamma_{\ell, k}, \quad k=0, \ldots, 2 \ell
$$

with

$$
\gamma_{\ell, k}=\frac{1}{2^{\ell-\zeta}}, \quad \zeta=\left\lfloor\frac{k+1}{2}\right\rfloor .
$$

Proof. From Proposition 1 and Theorem 4, for $i=0, \ldots, \ell$

$$
\left|T_{\ell}^{(2 i)}[f](x)\right|=\left|\int_{0}^{1} G_{\ell-i}(x, t) f^{(2 \ell+1)}(t) \mathrm{d} t\right| \leq M_{\ell} \int_{0}^{1}\left|G_{\ell-i}(x, t)\right| \mathrm{d} t,
$$


and for $i=0, \ldots, \ell-1$

$$
\left|T_{\ell}^{(2 i+1)}[f](x)\right|=\left|\int_{0}^{1} \frac{\partial}{\partial x} G_{\ell-i}(x, t) f^{(2 \ell+1)}(t) \mathrm{d} t\right| \leq M_{\ell} \int_{0}^{1}\left|\frac{\partial}{\partial x} G_{\ell-i}(x, t)\right| \mathrm{d} t .
$$

From the last two inequalities and Proposition 2, we have

$$
\begin{aligned}
& \left|T_{\ell}^{(2 i)}[f](x)\right| \leq M_{\ell} \frac{1}{2^{\ell-i}}, \quad i=0, \ldots, \ell, \\
& \left|T_{\ell}^{(2 i+1)}[f](x)\right| \leq M_{\ell} \frac{1}{2^{\ell-i+1}}, \quad i=0, \ldots, \ell-1 .
\end{aligned}
$$

Relations (2.27) and (2.28) can be written as (2.25).

Remark 3. We explicitly note that $\gamma_{\ell, 0}=\frac{1}{2^{\ell}}, \gamma_{\ell, 1}=\gamma_{\ell, 2}=\frac{1}{2^{\ell-1}}$.

From the previous inequalities, the following theorem can be proved.

Theorem 9. Let be $f \in C^{\infty}[0,1]$. Then, for a fixed $k$

$$
\lim _{\ell \rightarrow \infty} Q_{\ell}^{(k)}[f](x)=f^{(k)}(x)
$$

absolutely and uniformly in [0,1], providing that there exists a positive constant $\lambda$, with $|\lambda|<2$, and an integer $m$, such that $f^{(2 \ell+1)}=O\left(\lambda^{\ell-\zeta+1}\right)$, for all $\ell \geq m$ and $\zeta$ as in $(2.26)$.

Remark 4. We observe that the functions $\sin x$ and $\cos x$ satisfy Theorem 9 .

\section{The Second-Type Lidstone-Euler Boundary Value Problem}

In this section, we will investigate the existence and uniqueness of the solution of the second-type Lidstone-Euler BVP (1.1). As we said, to the best of the authors' knowledge, the existence and uniqueness of the solution of (1.1) have not previously been investigated. Similar BVPs with different boundary conditions have been much studied (see, for example, [1] and references therein).

If $f \equiv 0$, problem (1.1) has a unique solution $y(x)=Q_{r}(x)$, where $Q_{r}(x)$ is defined in (2.14).

The following theorem provides sufficient conditions for the existence and uniqueness of the solution of problem (1.1).

Theorem 10. Suppose that

(i) $k_{s}>0,0 \leq s \leq q$, are real given numbers and let $M$ be the maximum of $\left|f\left(x, y_{0}, \ldots, y_{q}\right)\right|$ on the compact set $[0,1] \times \Omega$, where

$$
\Omega=\left\{\left(y_{0}, \ldots, y_{q}\right)|| y_{s} \mid \leq 2 k_{s}, s=0, \ldots, q\right\} ;
$$

(ii) $C_{2 s}<k_{2 s}, C_{2 s+1}<k_{2 s+1}$, where $C_{2 s}$ and $C_{2 s+1}$ are defined in (2.18) and (2.19), respectively;

(iii) $\frac{M}{2^{r-s}}<k_{2 s}, \quad s=0, \ldots,\left\lfloor\frac{q}{2}\right\rfloor ; \quad \frac{M}{2^{r-s-1}}<k_{2 s+1}, \quad s=0, \ldots,\left\lfloor\frac{q-1}{2}\right\rfloor$; 
(iv) the function $f$ satisfies a uniform Lipschitz condition in $\left(y(x), y^{\prime}(x), \ldots\right.$, $\left.y^{(q)}(x)\right)$, that is, there exists a nonnegative constant $L$, such that the inequality

$$
\left|f\left(x, y_{0}, \ldots, y_{q}\right)-f\left(x, \bar{y}_{0}, \ldots, \bar{y}_{q}\right)\right| \leq L \sum_{k=0}^{q}\left|y_{k}-\bar{y}_{k}\right|
$$

holds whenever $\left(y_{0}, \ldots, y_{q}\right)$ and $\left(\bar{y}_{0}, \ldots, \bar{y}_{q}\right)$ belong to $\Omega$;

(v) $(q+1) D L<1$, where $D=\max _{0 \leq s \leq q}\left\{\max _{0 \leq x, t \leq 1}\left|\frac{\partial^{s}}{\partial x^{s}} K_{r}(x, t)\right|\right\}$.

Then, the boundary value problem (1.1) has a unique solution on $\Omega$.

Proof. It is known [16] that problem (1.1) is equivalent to the Fredholm integral equation

$$
\begin{aligned}
y(x) & =Q_{r}[y](x)+\int_{0}^{1} K_{r}(x, t) y^{(2 r+1)}(t) \mathrm{d} t \\
& =Q_{r}[y](x)+\int_{0}^{1} K_{r}(x, t) f\left(t, y(t), \ldots, y^{(q)}(t)\right),
\end{aligned}
$$

where $Q_{r}[y](x)$ is the polynomial defined in $(2.16)$ and $K_{r}(x, t)$ is the Peano kernel as in (2.24).

We define the operator $T: C^{q}[0,1] \rightarrow C^{2 r+1}[0,1]$ as follows:

$$
T[y](x):=Q_{r}[y](x)+\int_{0}^{1} K_{r}(x, t) f\left(t, y(t), \ldots, y^{(q)}(t)\right) \mathrm{d} t .
$$

Obviously, any fixed point of $T$ is a solution of the boundary value problem (1.1).

For all $y \in C^{q}[0,1]$, we introduce the norm $\|y\|=\max _{0 \leq s \leq q}\left\{\max _{0 \leq t \leq 1}\left|y^{(s)}(t)\right|\right\}$, so that $C^{q}[0,1]$ becomes a Banach space. Moreover, we consider the set

$$
B=\left\{y(t) \in C^{q}[0,1]\left|\max _{0 \leq t \leq 1}\right| y^{(s)}(t) \mid \leq 2 k_{s}, s=0, \ldots, q\right\} .
$$

The operator $T$ maps $B$ into itself. To show this, let $y(x) \in B$. Then

$$
(T[y])^{(2 s)}(x)=Q_{r}^{(2 s)}[y](x)+\int_{0}^{1} \frac{\partial^{2 s}}{\partial x^{2 s}}\left|K_{r}(x, t)\right| f\left(t, y(t), \ldots, y^{(q)}(t)\right) \mathrm{d} t .
$$

From hypotheses $(i)$ and $(i i i)$, and Propositions 1, 2, and 4 , we have that $T B \subseteq B$. In fact

$$
\begin{aligned}
\left|(T[y])^{(2 s)}(x)\right| & \leq C_{2 s}+M \int_{0}^{1}\left|K_{r-s}(x, t)\right| \mathrm{d} t \\
& \leq k_{2 s}+\frac{M}{2^{r-s}}<2 k_{2 s}, \quad s=0, \ldots,\left\lfloor\frac{q}{2}\right\rfloor,
\end{aligned}
$$


and

$$
\begin{aligned}
\left|(T[y])^{(2 s+1)}(x)\right| & \leq C_{2 s+1}+M \int_{0}^{1}\left|\frac{\partial}{\partial x} K_{r-s}(x, t)\right| \mathrm{d} t \\
& \leq k_{2 s+1}+\frac{M}{2^{r-s-1}}<2 k_{2 s+1}, \quad s=0, \ldots,\left\lfloor\frac{q-1}{2}\right\rfloor .
\end{aligned}
$$

From inequalities (3.2)-(3.3), we get that the sets $\left\{(T[y])^{(s)}(x) \mid y(x) \in\right.$ $B\}$ are uniformly bounded and equicontinuous in $[0,1]$, for all $0 \leq s \leq q$. From the Ascoli-Arzela theorem, this implies that $\overline{T B}$ is compact. Hence, from the Shauder fixed point theorem, there exists a fixed point of $T$ in $\Omega$.

Now, we will prove the uniqueness. Suppose that there exist two distinct solutions $y(x), z(x)$ of problem (1.1). It results

$$
\begin{aligned}
y^{(s)} & (x)-z^{(s)}(x) \\
= & \int_{0}^{1} \frac{\partial^{s}}{\partial x^{s}} K_{r}(x, t)\left[f\left(t, y(t), y^{\prime}(t), \ldots, y^{(q)}(t)\right)\right. \\
& \left.-f\left(t, z(t), z^{\prime}(t), \ldots, z^{(q)}(t)\right)\right] \mathrm{d} t,
\end{aligned}
$$

$s=0, \ldots, q$. Hence

$$
\begin{aligned}
\left|y^{(s)}(x)-z^{(s)}(x)\right| & \leq \max _{0 \leq s \leq q}\left\{\max _{0 \leq x, t \leq 1}\left|\frac{\partial^{s}}{\partial x^{s}} K_{r}(x, t)\right|\right\} L \sum_{i=0}^{q} \int_{0}^{1}\left|y^{(i)}(t)-z^{(i)}(t)\right| \mathrm{d} t \\
& \leq D L \sum_{i=0}^{q} \int_{0}^{1} \max _{0 \leq i \leq q}\left\{\max _{0 \leq t \leq 1}\left|y^{(i)}(t)-z^{(i)}(t)\right|\right\} \mathrm{d} t
\end{aligned}
$$

so that

$$
\|y-z\| \leq(q+1) D L\|y-z\| .
$$

From hypothesis $(v)$, the uniqueness of the solution follows.

\section{Computational Aspects}

For the numerical solution of high odd-order boundary value problems, many approaches have been proposed such as: spline interpolation [9,30], nonpolynomial spline techniques [29], Galerkin methods [17], variational iterative techniques [31,33], and modified decomposition method [35]. In the following, we present two numerical approaches: Bernstein extrapolation and collocation methods.

\subsection{Bernstein Extrapolation Methods}

The first numerical approach for the numerical solution of the BVP (1.1) is based on extrapolated Bernstein polynomials [5]. 
We recall that, given a real function $g(x)$ defined in $[0,1]$, the $n-t h$ Bernstein polynomial for $g$ is given by

$$
B_{n}[g](x)=\sum_{k=0}^{n} b_{n, k}(x) g\left(\frac{k}{n}\right), \quad b_{n, k}(x)=\left(\begin{array}{l}
k \\
n
\end{array}\right) x^{k}(1-x)^{n-k} .
$$

Then, it is known the following

Theorem 11. [18] Let $g$ be a bounded real function in $I=[0,1]$. Then

$$
\lim _{n \rightarrow \infty} B_{n}[g](x)=g(x),
$$

at any point $x \in I$ at which $g$ is continuous, and, if we pose $\bar{R}_{n}[g](x)=$ $B_{n}[g](x)-g(x)$,

$$
\left|\bar{R}_{n}[g](x)\right| \leq \frac{5}{4} \omega\left(g ; n^{-\frac{1}{2}}\right),
$$

where $\omega$ is the modulus of continuity of $g$ on I. If $g \in C(I)$, the convergence is uniform in $I$.

Moreover, if $g$ is twice differentiable in $I$, then

$$
\lim _{n \rightarrow \infty} n\left[g(x)-B_{n}[g](x)\right]=\frac{x(1-x)}{2} g^{\prime \prime}(x) .
$$

Theorem 12. [5] If $g \in C^{2 s}(I), s \geq 1$, then the Bernestein polynomial for $g$ has the following asymptotic expansion:

$$
B_{n}[g](x)=g(x)+\sum_{i=1}^{s} h^{i} S_{i}[g](x)+h^{s+1} E_{h}[g](x),
$$

where $h=\frac{1}{n}$, the functions $S_{i}[g](x), i=0, \ldots, s$, do not depend on $h$ and $E_{h}[g](x) \rightarrow 0$ for $h \rightarrow 0$.

From Theorems 11 and 12, we can prove the following theorem.

Theorem 13. Let $y(x)$ be the solution of problem (1.1). Then

$$
y(x)=Q_{r}[y](x)+\sum_{k=0}^{n} p_{n, k}(x) f\left(x_{k}, y\left(x_{k}\right), \ldots, y^{(q)}\left(x_{k}\right)\right)+R_{n}[y](x),
$$

where $x_{k}=\frac{k}{n}$

$$
\begin{aligned}
& p_{n, k}(x)=\int_{0}^{1} K_{r}(x, t) b_{n, k}(t) \mathrm{d} t, \quad k=0, \ldots, n \\
& \left|R_{n}[y](x)\right| \leq \frac{5}{2^{r+2}} \omega\left(y^{(2 r+1)} ; n^{-\frac{1}{2}}\right)
\end{aligned}
$$

Moreover, if $y \in C^{2 r+3}(I)$, then

$$
\left|R_{n}[y](x)\right| \leq \frac{1}{2^{r+3} n} \max _{0 \leq x \leq 1}\left|y^{(2 r+3)}(x)\right|,
$$

and the convergence is uniform. 
Proof. If $y(x)$ is the solution of problem (1.1), we get relation (3.1). The thesis follows after easy calculations from Theorem 12 if $g \equiv y^{(2 r+1)}$.

The proposed method for the numerical solution of problem (1.1) is based on the results of the previous theorems.

$\forall n \in \mathbb{N}$, let us set

$$
\phi_{n}(x)=Q_{r}[y](x)+\sum_{i=0}^{n} p_{n, i}(x) f\left(x_{i}, y\left(x_{i}\right), \ldots, y^{(q)}\left(x_{i}\right)\right), \quad x_{i}=\frac{i}{n} \text {. }
$$

Corollary 3. For the solution $y(x)$ of problem (1.1), we get

$$
\lim _{n \rightarrow \infty} \phi_{n}(x)=y(x),
$$

uniformly in $x \in I$. Moreover

$$
\left\|y-\phi_{n}\right\|=\left\|R_{n}[y]\right\| \leq \frac{5}{2^{r+2}} \omega\left(y^{(2 r+1)} ; n^{-\frac{1}{2}}\right) .
$$

We call $\phi_{n}(x)$ the approximating solution of first order, and the error is bounded by (4.3).

To have approximating functions of higher order, we use the following asymptotic expansion.

Theorem 14. Let $n, m$ be two positive integers and $h=\frac{1}{n}$. Moreover, let $y(x) \in C^{2(r+m+1)}(I)$ be the solution of problem (1.1). Then

$$
y(x)=\phi_{n}(x)+\sum_{i=0}^{m} h^{i} \bar{S}_{i}[y](x)+h^{m+1} \bar{E}_{h}[y](x),
$$

where the functions $\bar{S}_{i}[y](x)$ do not depend on $h$, and $\bar{E}_{h}[y](x) \rightarrow 0$ for $h \rightarrow 0$.

Proof. The proof follows after easy calculations by applying Theorem 12 at relation (3.1).

The expansion (4.4) in Theorem 14 suggests to apply the extrapolation procedure $[5,32]$ described in the following Theorem.

Theorem 15. Let $y \in C^{2(r+m)}[0,1]$, with $m$ a fixed positive integer. Let $\left\{n_{k}\right\}_{k}$ be an increasing sequence of positive integers and $h_{k}=n_{k}^{-1}$. We define a sequence of polynomials of degree $n_{i+k}$ as follows:

$$
\begin{cases}T_{0}^{(i)}:=T_{0}^{(i)}[y](x)=\phi_{n_{i}}(x), & i=0, \ldots, m \\ T_{k}^{(i)}:=T_{k}^{(i)}[y](x)=\frac{h_{i+k} T_{k-1}^{(i)}-h_{i} T_{k-1}^{(i+1)}}{h_{i+k}-h_{i}}, & k=1, \ldots, m-1 \\ & i=0, \ldots, m-k\end{cases}
$$

Then, for $i=0, \ldots, m-k$

$$
\lim _{h_{i} \rightarrow 0} T_{k}^{(i)}=y(x), \quad k=1,2, \ldots, m-1 .
$$


Moreover, the following representations of the error and of $T_{k}^{(i)}$ hold:

$$
\begin{aligned}
& T_{k}^{(i)}-y(x)=(-1)^{k} h_{i} h_{i+1} \cdots h_{k}\left(\bar{S}_{k+1}[y](x)+O\left(h_{i}\right)\right), \\
& T_{k}^{(i)}[y](x)=\sum_{j=0}^{k} l_{j}(0) y_{n_{j}}(x), \quad l_{j}(h)=\prod_{i=0, i \neq j}^{k} \frac{h_{i}-h}{h_{i}-h_{j}} .
\end{aligned}
$$

From Theorem 15, for any $z \in[0,1], y(z)$ is approximated by $T_{m}^{(0)}[y](z)$, $n_{m}$ being the last element of the considered numerical sequence $\left\{n_{i}\right\}_{i}$.

\section{Algorithm for Practical Calculations}

To calculate the first-order approximation $\phi_{n}(x)$ by formula (4.2), we need the values $y_{i}^{(s)} \approx y^{(s)}\left(x_{i}\right), s=0, \ldots, q, i=0, \ldots, n$, with $s \neq 2 j+1, j=$ $0, \ldots,\left\lfloor\frac{q-1}{2}\right\rfloor$, when $i=0$ and $s \neq 2 j, j=0, \ldots,\left\lfloor\frac{q}{2}\right\rfloor$, when $i=n$.

To this aim, we consider the algebraic system of dimension $n(q+1)$

$$
y_{i}^{(s)}=Q_{r}^{(s)}[y]\left(x_{i}\right)+\sum_{k=0}^{n} p_{n, k}^{(s)}\left(x_{i}\right) f\left(x_{k}, y_{k}, y_{k}^{\prime} \ldots, y_{k}^{(q)}\right), \quad \begin{aligned}
& i=0, \ldots, n, \\
& s=0, \ldots, q,
\end{aligned}
$$

and $y_{0}^{(2 j+1)}=y^{(2 j+1)}(0)=\alpha_{j}, j=0, \ldots,\left\lfloor\frac{q-1}{2}\right\rfloor, y_{n}^{(2 j)}=y^{(2 j)}(1)=\beta_{j}$, $j=0, \ldots,\left\lfloor\frac{q}{2}\right\rfloor$.

Let us put $Y_{n}^{q}=\left(\bar{Y}_{0}, \ldots, \bar{Y}_{q}\right)^{T}$, with $\bar{Y}_{2 j}=\left(y_{0}^{(2 j)}, \ldots, y_{n-1}^{(2 j)}\right), j=$ $0, \ldots,\left\lfloor\frac{q}{2}\right\rfloor, \bar{Y}_{2 j+1}=\left(y_{1}^{(2 j+1)}, \ldots, y_{n}^{(2 j+1)}\right), j=0, \ldots,\left\lfloor\frac{q-1}{2}\right\rfloor$

$$
A_{n}^{q}=\left(\begin{array}{cccc}
A_{0} & 0 & \cdots & 0 \\
0 & \ddots & & \vdots \\
\vdots & & \ddots & 0 \\
0 & \cdots & 0 & A_{q}
\end{array}\right) \text {, }
$$

with $A_{j} \in \mathbb{R}^{n \times(n+1)}$

$$
\begin{aligned}
& A_{2 j}=\left(\begin{array}{ccc}
p_{n, 0}^{(2 j)}\left(x_{0}\right) & \cdots & p_{n, n}^{(2 j)}\left(x_{0}\right) \\
\vdots & \vdots \\
p_{n, 0}^{(2 j)}\left(x_{n-1}\right) & \cdots & p_{n, n}^{(2 j)}\left(x_{n-1}\right)
\end{array}\right), \quad j=0, \ldots,\left\lfloor\frac{q}{2}\right\rfloor, \\
& A_{2 j+1}=\left(\begin{array}{ccc}
p_{n, 0}^{(2 j+1)}\left(x_{1}\right) & \cdots & p_{n, n}^{(2 j+1)}\left(x_{1}\right) \\
\vdots & \vdots \\
p_{n, 0}^{(2 j+1)}\left(x_{n}\right) & \cdots & p_{n, n}^{(2 j+1)}\left(x_{n}\right)
\end{array}\right), \quad j=0, \ldots,\left\lfloor\frac{q-1}{2}\right\rfloor .
\end{aligned}
$$

Moreover

$$
F_{Y_{n}^{q}}=(\underbrace{F_{n}, \ldots, F_{n}}_{q+1})^{T}
$$


with

$$
F_{n}=\left(f_{0}, \ldots, f_{n}\right)^{T}, \quad f_{k}=f\left(x_{k}, y_{k}, y_{k}^{\prime} \ldots, y_{k}^{(q)}\right), \quad k=0, \ldots, n
$$

and

$$
C_{n}^{q}=\left(C_{0}, \ldots, C_{q}\right)^{T}
$$

with

$$
\begin{gathered}
C_{2 j}=\left(Q_{r}^{(2 j)}[y]\left(x_{0}\right), \ldots, Q_{r}^{(2 j)}[y]\left(x_{n-1}\right)\right), \quad j=0, \ldots,\left\lfloor\frac{q}{2}\right\rfloor, \\
C_{2 j+1}=\left(Q_{r}^{(2 j+1)}[y]\left(x_{1}\right), \ldots, Q_{r}^{(2 j+1)}[y]\left(x_{n}\right)\right), \quad j=0, \ldots,\left\lfloor\frac{q-1}{2}\right\rfloor .
\end{gathered}
$$

Thus, system (5.1) can be written in the form

$$
Y_{n}^{q}-A_{n}^{q} F_{Y_{n}^{q}}=C_{n}^{q}
$$

or

$$
Y_{n}^{q}=G\left(Y_{n}^{q}\right), \quad \text { with } \quad G\left(Y_{n}^{q}\right)=A F_{Y_{n}^{q}}+C_{n}^{q} .
$$

Proposition 5. For the matrix $A_{n}^{q}$, the following relation holds:

$$
\left\|A_{n}^{q}\right\|_{\infty}=O(1), \quad n \rightarrow \infty \text {. }
$$

Proof. $\left\|A_{n}^{q}\right\|_{\infty}=\max _{0 \leq s \leq q}\left\|A_{s}\right\|_{\infty}$. Since all Bernstein basis functions $b_{n, k}(t)$ of the same order have the same definite integral over the interval $[0,1]$, that is $\int_{0}^{1} b_{n, k}(t) \mathrm{d} t=\frac{1}{n+1}$, we have that

- if $s=2 j, j=0, \ldots,\left\lfloor\frac{q}{2}\right\rfloor$, then

$$
\begin{aligned}
\left|p_{n, k}^{(2 j)}\left(x_{i-1}\right)\right| & \leq \int_{0}^{1}\left|\frac{\partial^{2 j}}{\partial x^{2 j}} K_{r}(x, t)\right|_{x=x_{i-1}} b_{n, k}(t) \mathrm{d} t \\
& =\int_{0}^{1}\left|K_{r-j}\left(x_{i-1}, t\right)\right| b_{n, k}(t) \mathrm{d} t \leq \frac{1}{n+1}\left|K_{r-j}\left(x_{i-1}, \bar{t}\right)\right|, \quad \bar{t} \in(0,1) ;
\end{aligned}
$$

hence

$$
\begin{aligned}
\left\|A_{s}\right\|_{\infty} & =\max _{0 \leq i \leq n} \sum_{k=0}^{n}\left|p_{n, k}^{(2 j)}\left(x_{i-1}\right)\right| \leq \frac{1}{n+1} \sum_{k=0}^{n} \max _{0 \leq i \leq n}\left|K_{r-j}\left(x_{i-1}, \bar{t}\right)\right| \\
& =\max _{0 \leq i \leq n}\left|K_{r-j}\left(x_{i-1}, \bar{t}\right)\right|
\end{aligned}
$$

- if $s=2 j+1, j=0, \ldots,\left\lfloor\frac{q-1}{2}\right\rfloor$, then

$$
\begin{aligned}
& \left|p_{n, k}^{(2 j+1)}\left(x_{i}\right)\right| \leq \int_{0}^{1}\left|\frac{\partial^{2 j+1}}{\partial x^{2 j+1}} K_{r}(x, t)\right|_{x=x_{i-1}} b_{n, k}(t) \mathrm{d} t \\
& =\int_{0}^{1}\left|\frac{\partial}{\partial x} K_{r-j}(x, t)\right|_{x=x_{i-1}} b_{n, k}(t) \mathrm{d} t \leq \frac{1}{n+1}\left|\frac{\partial}{\partial x} K_{r-j}\left(x_{i}, \tilde{t}\right)\right|, \quad \tilde{t} \in(0,1) ;
\end{aligned}
$$

hence

$$
\begin{aligned}
\left\|A_{s}\right\|_{\infty} & =\max _{0 \leq i \leq n} \sum_{k=0}^{n}\left|p_{n, k}^{(2 j+1)}\left(x_{i}\right)\right| \leq \frac{1}{n+1} \sum_{k=0}^{n} \max _{0 \leq i \leq n}\left|\frac{\partial}{\partial x} K_{r-j}\left(x_{i}, \tilde{t}\right)\right| \\
& =\max _{0 \leq i \leq n}\left|\frac{\partial}{\partial x} K_{r-j}\left(x_{i}, \tilde{t}\right)\right| .
\end{aligned}
$$


From definition of $K_{l}(x, t)$, there exist $M_{1}, M_{2} \in \mathbb{R}$, such that $\left|K_{l}(x, t)\right| \leq M_{1}$ and $\left|\frac{\partial}{\partial x} K_{l}(x, t)\right| \leq M_{2}, l \geq 0$, for all $0 \leq x, t \leq 1$. From this, the result follows.

Lemma 1. With the previous notations and hypothesis, system (5.2) has a unique solution if $T=L\|A\|_{\infty}<1$. The solution can be calculated by the iterations

$$
\left(Y_{n}^{q}\right)_{j+1}=G\left(\left(Y_{n}^{q}\right)_{j}\right), \quad j=0, \ldots, N
$$

with a fixed $\left(Y_{n}\right)_{0} \in \mathbb{R}^{n(q+1)}$. Moreover, at the $j$ th iteration, the error is

$$
\left\|\left(Y_{n}^{q}\right)_{j+1}-Y_{n}^{q}\right\|_{\infty} \leq \frac{T^{j}}{1-T}\left\|\left(Y_{n}^{q}\right)_{1}-\left(Y_{n}^{q}\right)_{0}\right\|_{\infty} .
$$

Proof. The proof follows by standard technique by applying the well-known contraction principle and Proposition 5.

The previous Lemma allows us to consider the first-order approximating function

$$
\bar{\phi}_{n}(x)=Q_{r}[y](x)+\sum_{k=0}^{n} p_{n, k}(x) f\left(x_{k}, y_{k}, y_{k}^{\prime} \ldots, y_{k}^{(q)}\right) .
$$

Proposition 6. Let $y(x)$ be the solution of problem (1.1) and $\bar{\phi}_{n}(x)$ the firstorder approximation. Then

$$
\left\|y-\bar{\phi}_{n}\right\|=o(1)
$$

if $L Z<1$, where $Z=(q+1) D$ with $D$ as in Theorem 10 .

Proof. For all $j=0, \ldots, q$ and for all $x \in[0,1]$, from (4.2), we get

$$
\phi_{n}^{(j)}(x)=Q_{r}^{(j)}[y](x)+\sum_{k=0}^{n} p_{n, k}^{(j)}(x) f\left(x_{k}, y\left(x_{k}\right), y^{\prime}\left(x_{k}\right) \ldots, y\left(x_{k}\right)^{(q)}\right),
$$

and by differentiating the (5.3)

$$
\bar{\phi}_{n}^{(j)}(x)=Q_{r}^{(j)}[y](x)+\sum_{k=0}^{n} p_{n, k}^{(j)}(x) f\left(x_{k}, y_{k}, y_{k}^{\prime} \ldots, y_{k}^{(q)}\right) .
$$

From (5.4), (5.5) and (4.1), we obtain

$$
\begin{aligned}
\phi_{n}^{(j)}(x)-\bar{\phi}_{n}^{(j)}(x)= & \sum_{k=0}^{n}\left[f\left(x_{k}, y\left(x_{k}\right), y^{\prime}\left(x_{k}\right) \ldots, y^{(q)}\left(x_{k}\right)\right)-f\left(x_{k}, y_{k}, y_{k}^{\prime}, \ldots, y_{k}^{(q)}\right)\right] \\
& \times \int_{0}^{1} \frac{\partial^{j}}{\partial x^{j}} K_{r}(x, t) b_{n, k}(t) \mathrm{d} t .
\end{aligned}
$$


Hence, from the Lipschitz property of $f$

$$
\begin{aligned}
& \left|\phi_{n}^{(j)}(x)-\bar{\phi}_{n}^{(j)}(x)\right| \leq \sum_{k=0}^{n}\left(L \sum_{i=0}^{q}\left|y^{(i)}\left(x_{k}\right)-y_{k}^{(i)}\right|\right) \max _{0 \leq x, t \leq 1}\left|\frac{\partial^{j}}{\partial x^{j}} K_{r}(x, t)\right| \frac{1}{n+1} \\
& \quad \leq L \max _{0 \leq i \leq q}\left\{\max _{0 \leq k \leq q}\left|y^{(i)}\left(x_{k}\right)-y_{k}^{(i)}\right|\right\}(q+1) \max _{0 \leq x, t \leq 1}\left|\frac{\partial^{j}}{\partial x^{j}} K_{r}(x, t)\right| \\
& \quad \leq L \max _{0 \leq i \leq q}\left\{\max _{0 \leq x \leq 1}\left|y^{(i)}(x)-\bar{\phi}_{n}^{(i)}(x)\right|\right\}(q+1) \max _{0 \leq j \leq q}\left\{\max _{0 \leq x, t \leq 1}\left|\frac{\partial^{j}}{\partial x^{j}} K_{r}(x, t)\right|\right\} \\
& \quad=L Z\left\|y-\bar{\phi}_{n}\right\| .
\end{aligned}
$$

Finally, the last inequality yields

$$
\left\|y-\bar{\phi}_{n}\right\| \leq\left\|y-\phi_{n}\right\|+\left\|\phi_{n}-\bar{\phi}_{n}\right\| \leq\left\|y-\phi_{n}\right\|+L Z\left\|y-\bar{\phi}_{n}\right\| ;
$$

hence, the thesis follows, according to Corollary 3.

The Bernstein extrapolation method for BVP (1.1) can be summarized as follows:

1. Data input: problem (1.1);

2. for a fixed $n \in \mathbb{N}$, solve the algebraic system (5.1);

3. for a fixed $m \in \mathbb{N}$, choose a sequence $n_{i}, i=0, \ldots, m$ and calculate $\bar{\phi}_{n_{i}}(x)$;

4. for $k=1, \ldots, m-1, i=0, \ldots, m-k$, calculate $T_{k}^{(i)}$.

\subsection{Collocation-Birkhoff-Lagrange Approach}

The collocation-Birkhoff-Lagrange approach to BVPs has been proposed in [16]. Here, we use this approach as comparisons with the method described above.

Let $y(x)$ be the solution of problem (1.1). For any $n \in \mathbb{N}$, if $y(x) \in$ $C^{2 r+n+2}[0,1]$, we can approximate $y^{(2 r+1)}(x)$ in $x \in[0,1]$ by the well-known Lagrange interpolation polynomial

$$
y^{(2 r+1)}(x)=L_{n}[y](x)+R_{n}[y](x),
$$

where

$$
L_{n}[y](x)=\sum_{i=0}^{n} l_{i}(x) y^{(2 r+1)}\left(x_{i}\right), \quad R_{n}[y](x)=\frac{1}{(n+1) !} \omega_{n}(x) y^{(2 r+n+2)}\left(\xi_{x}\right),
$$

$l_{i}(x)$ being the fundamental Lagrange polynomials and $\omega_{n}(x)=\prod_{i=0}^{n}\left(x-x_{i}\right)$, with $x_{i}$, for $i=0 \ldots, n, n+1$ distinct points in $[0,1]$ and $\xi_{x}$ a point in the smallest interval containing $x$ and all $x_{i}, i=0, \ldots, n$.

Then, by substituting (5.6) in (3.1), we have

$$
y(x)=Q_{r}[y](x)+\sum_{i=0}^{n} q_{n, i}(x) f\left(x_{i}, y\left(x_{i}\right), y^{\prime}\left(x_{i}\right), \ldots, y^{(q)}\left(x_{i}\right)\right)+T_{r, n}[y](x),
$$

where

$$
q_{n, i}(x)=\int_{0}^{1} K_{r}(x, t) l_{i}(t) \mathrm{d} t, \quad i=0, \ldots, n
$$


and the remainder term $T_{r, n}[y](y, x)$ is given by

$$
T_{r, n}[y](x)=\frac{1}{(n+1) !} \int_{0}^{1} K_{r}(x, t) \omega_{n}(t) y^{(2 r+n+2)}\left(\xi_{t}\right) \mathrm{d} t .
$$

Relation (5.7) suggests to consider the implicitly defined polynomial

$$
y_{n}(x)=Q_{r}[y](x)+\sum_{i=0}^{n} q_{n, i}(x) f\left(x_{i}, y_{n}\left(x_{i}\right), y_{n}^{\prime}\left(x_{i}\right), \ldots, y_{n}^{(q)}\left(x_{i}\right)\right) \text {. }
$$

Theorem 16. [16] The polynomial $y_{n}(x)$ of degree $2 r+n+1$ defined in (5.8) is a collocation polynomial for (1.1) at nodes $x_{i}$.

An algorithm for practical calculation is similar to that used in [16].

\section{Numerical Examples}

As we said, there are no specific methods for the numerical solution of problem (1.1). Neither specific numerical examples we have found in the literature, to compare the numerical results. Anyhow, in the following, we report some problems to validate the theoretical results previously given. Since the analytical solutions of the considered examples are known, we compute the true errors $\forall x \in I$ fixed

$e_{B, n}(x)=\left|y(x)-\bar{\phi}_{n}(x)\right|, \quad E_{B, m}(x)=\left|y(x)-T_{m}^{(0)}[y](x)\right|, \quad e_{L, n}(x)=\left|y(x)-\bar{y}_{n}(x)\right|$.

Example 1. Consider the following problem:

$$
\left\{\begin{array}{l}
y^{\prime \prime \prime}(x)+2 e^{-3 y(x)}=4(1+x)^{-3}, \quad x \in[0,1] \\
y(1)=\log 2, \quad y^{\prime}(0)=1, \\
y^{\prime \prime}(1)=-\frac{1}{4} .
\end{array}\right.
$$

The analytical solution is $y(x)=\log (1+x)$.

The first approximating polynomials $\bar{\phi}_{n}(x)$ are

$$
\begin{aligned}
\bar{\phi}_{2}(x)= & 0.10803+x-0.70961 x^{2}+0.42560 x^{3}-0.15413 x^{4}+0.023259 x^{5} \\
\bar{\phi}_{3}(x)= & 0.06454+x-0.62952 x^{2}+0.39201 x^{3}-0.17309 x^{4}+0.04420 x^{5}-0.00500 x^{6} \\
\bar{\phi}_{4}(x)= & 0.04531+x-0.59258 x^{2}+0.37570 x^{3}-0.18558 x^{4}+0.06144 x^{5}-0.01226 x^{6} \\
& +0.00112 x^{7} \\
\bar{\phi}_{5}(x)= & 0.03473+x-0.57175 x^{2}+0.36631 x^{3}-0.19457 x^{4}+0.07557 x^{5}-0.02022 x^{6} \\
& +0.00333 x^{7}-0.00025 x^{8} \\
\bar{\phi}_{6}(x)= & 0.02808+x-0.55847 x^{2}+0.36027 x^{3}-0.20137 x^{4}+0.08726 x^{5}-0.02815 x^{6} \\
& +0.00636 x^{7}-0.00089 x^{8}+0.00006 x^{9} .
\end{aligned}
$$

Figure 1 shows the graphs of the error functions $e_{B, n_{k}}(x)$ with $n_{k}=4+2 k$, $k=0, \ldots, 3$ (Fig. 1a) and the graph of $E_{B, 3}(x)$ (Fig. 1b). Figure 1a shows the low convergence of the approximating polynomial sequence $\left\{\bar{\phi}_{n}\right\}$.

The absolute errors in $x=\frac{1}{2}$ using extrapolation for different sequences $n_{k}, k=0, \ldots, m$, are displayed in Table 1 . 


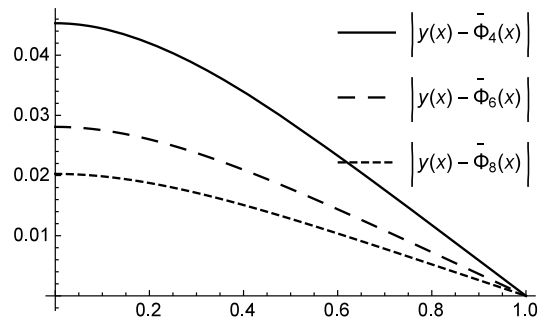

(a) $e_{B, n_{k}}(x)$

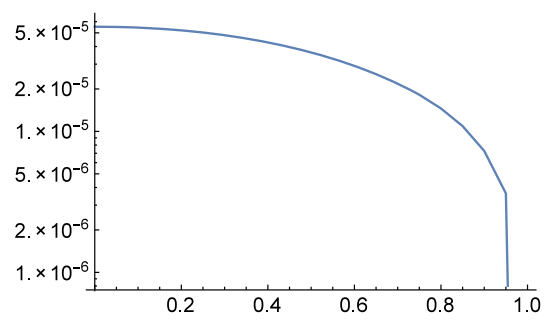

(b) $E_{B, 3}(x)$

Figure 1. Error functions for $n_{k}=4+2 k, k=0, \ldots, 3$ Problem (6.1)

Figure 2 shows the graphs of the error functions $e_{L, n}(x)$ in the case of collocation on equidistant nodes, for several values of the degree $n$ of the approximating polynomials.

Table 2 lists the comparison between the approximation by extrapolated Bernstein polynomials (using one of the sequences considered in Table 1) and collocation-Birkhoff-Lagrange polynomials of degree 10 and 12, respectively.

Example 2. Consider the following problem:

$$
\left\{\begin{array}{l}
y^{(v)}(x)=y^{2}(x) e^{-x}, \quad x \in[0,1] \\
y(1)=y^{\prime \prime}(1)=y^{(i v)}(1)=e, \\
y^{\prime}(0)=y^{\prime \prime \prime}(0)=1 .
\end{array}\right.
$$

The analytical solution is $y(x)=e^{x}$.

Figure 3 shows the graphs of the error functions $e_{B, n_{k}}(x)$ with $n_{k}=$ $2+2 k, k=0, \ldots, 3$ (Fig. 3a) and the graph of $E_{B, 2}(x)$ (Fig. 3b).

The errors in $x=\frac{1}{2}$ using extrapolation for different sequences $n_{k}$ are displayed in Table 3.

Figure 4 shows the graphs of the error functions $e_{L, n}(x)$ in the case of collocation on equidistant nodes, for several values of the degree $n$ of the approximating polynomials.

Table 1. Extrapolation error in $x=\frac{1}{2}-$ Problem (6.1)

\begin{tabular}{lll}
\hline$n_{k}$ & $m$ & $E_{B, m}\left(\frac{1}{2}\right)$ \\
\hline $2^{k+2}$ & 2 & $2.2214 e-05$ \\
$4+2 k$ & 3 & $1.1868 e-05$ \\
& 5 & $1.9840 e-07$ \\
$10+5 k$ & 6 & $1.8918 e-09$ \\
& 2 & $4.3119 e-06$ \\
$2,3,4,6,8$ & 3 & $6.6589 e-07$ \\
$2,3,4,6,8,12$ & 4 & $1.3789 e-05$ \\
\hline
\end{tabular}



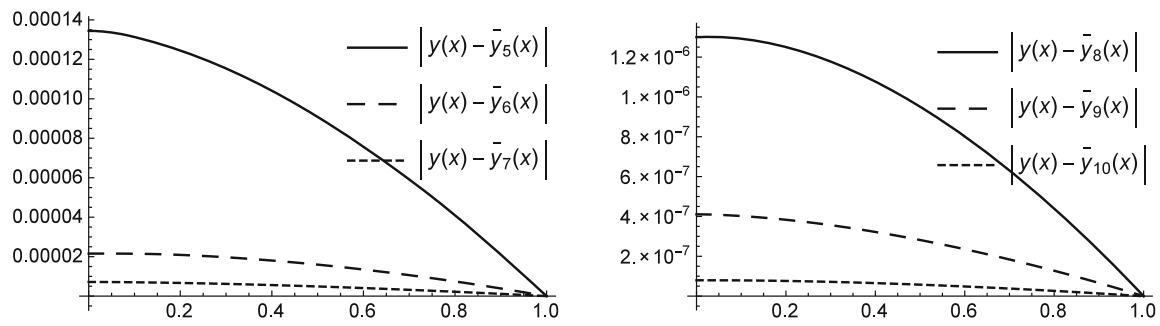

Figure 2. Error functions $e_{L, n}(x)$, Problem (6.1)

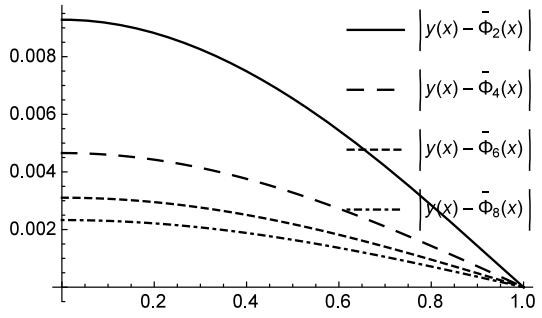

(a) $e_{B, n_{k}}(x)$

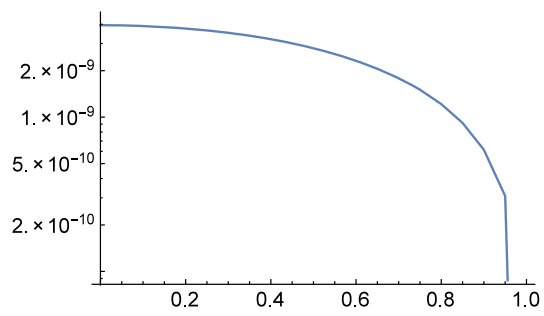

(b) $E_{B, 3}(x)$

Figure 3. Error functions for $n_{k}=2+2 k, k=0, \ldots, 3$ Problem (6.2)

Table 4 lists the comparison between the approximation by extrapolated Bernstein polynomials and collocation-Birkhoff-Lagrange polynomials.

\section{Conclusions}

In this paper, we considered general nonlinear high odd-order differential equations with Lidstone-Euler boundary conditions of second type. First, we studied the associated interpolation problem and we obtained new properties, such as the integral Cauchy and Peano representation of the error, bounds for the error, and its derivatives, and we deducted the interesting convergence properties of the interpolatory polynomial sequences. Then, we

Table 2. Comparison between extrapolated Bernstein polynomials and collocation-Birkhoff-Lagrange polynomialsProblem (6.1)

\begin{tabular}{|c|c|c|c|}
\hline \multicolumn{2}{|l|}{$\left\|E_{B, m}\right\|$} & \multicolumn{2}{|l|}{$\left\|e_{L, n}\right\|$} \\
\hline$n_{k}=4+2 k$ & & & \\
\hline$m=5$ & $m=6$ & $n=10$ & $n=12$ \\
\hline $2.9285 e-07$ & $7.2597 e-09$ & $7.9449 e-08$ & $7.035 e-09$ \\
\hline
\end{tabular}


Table 3. Extrapolation error in $x=\frac{1}{2}$-Problem (6.2)

\begin{tabular}{lll}
\hline$n_{k}$ & $m$ & $E_{B, m}\left(\frac{1}{2}\right)$ \\
\hline $4+k$ & 3 & $3.5572 e-08$ \\
$4+2 k$ & 5 & $9.5277 e-12$ \\
& 3 & $1.4912 e-08$ \\
$2+2 k$ & 4 & $4.6073 e-10$ \\
& 4 & $2.7929 e-09$ \\
$2,3,4,6,8$ & 5 & $5.7065 e-12$ \\
$2,3,4,6,8,12$ & 4 & $9.4358 e-09$ \\
\hline
\end{tabular}
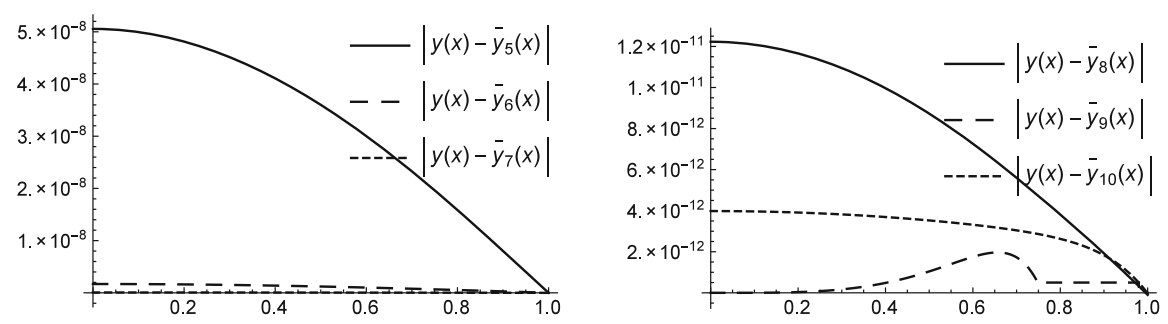

Figure 4. Error functions $e_{L, n}(x)$, Problem (6.2)

Table 4. Comparison between extrapolated Bernstein polynomials and collocation-Birkhoff-Lagrange polynomialsProblem (6.2)

\begin{tabular}{|c|c|c|c|}
\hline \multicolumn{2}{|l|}{$\left\|E_{B, m}\right\|$} & \multicolumn{2}{|l|}{$\left\|e_{L, n}\right\|$} \\
\hline$n_{k}=2+2 k$ & & & \\
\hline $\bar{m}=4$ & $m=5$ & $n=7$ & $n=9$ \\
\hline $3.9448 e-09$ & $9.9367 e-12$ & $6.6291 e-11$ & $2.2598 e-11$ \\
\hline
\end{tabular}

considered the associated Lidstone-Euler second-type boundary value problem, from both a theoretical and a computational point of view. Particularly, we gave a theorem of existence and uniqueness of the solution of the problem and we proposed two different numerical approaches for the approximate solution: one of them is based on extrapolated Bernstein polynomials, the other one on Lagrange interpolation and collocation principle. We note the convergence properties of the Bernstein extrapolation method and, in some cases, a greater computational accuracy of the Lagrange-collocation methods. From the numerical examples, we can observe that the order of the error is similar with the two different methods. Further developments are possible and desirable as well theoretical as well computational. 


\section{Acknowledgements}

One of the authors would like to thank the support of INdAM-GNCS Project 2020 .

Funding Open access funding provided by Universitá della Calabria within the CRUI-CARE Agreement.

Open Access. This article is licensed under a Creative Commons Attribution 4.0 International License, which permits use, sharing, adaptation, distribution and reproduction in any medium or format, as long as you give appropriate credit to the original author(s) and the source, provide a link to the Creative Commons licence, and indicate if changes were made. The images or other third party material in this article are included in the article's Creative Commons licence, unless indicated otherwise in a credit line to the material. If material is not included in the article's Creative Commons licence and your intended use is not permitted by statutory regulation or exceeds the permitted use, you will need to obtain permission directly from the copyright holder. To view a copy of this licence, visit http:// creativecommons.org/licenses/by/4.0/.

Publisher's Note Springer Nature remains neutral with regard to jurisdictional claims in published maps and institutional affiliations.

\section{References}

[1] Agarwal, R.P.: Boundary Value Problems for Higher Order Differential Equations. World Scientific Publishing Co. Inc, Teaneck (1986)

[2] Akram, G., Sadaf, M.: Application of homotopy analysis method to the solution of ninth order boundary value problems in AFTI-F16 fighters. J. Assoc. Arab Univ. Basic Appl. Sci. 24, 149-155 (2017)

[3] Bhrawy, A.H., Abd-Elhameed, W.M.: New algorithm for the numerical solutions of nonlinear third-order differential equations using Jacobi-Gauss collocation method. Math. Prob. Eng. 2011, 837218 (2011)

[4] Chandrasekhar, S.: Hydrodynamic and Hydromagnetic Stability. Clarendon, International Series of Monographs on Physics, Oxford (1961)

[5] Costabile, F., Gualtieri, M.I., Serra, S.: Asymptotic expansion and extrapolation for Bernstein polynomials with applications. BIT Numer. Math. 36(4), 676-687 (1996)

[6] Costabile, F., Napoli, A.: A method for high-order multipoint boundary value problems with Birkhoff-type conditions. Int. J. Comput. Math. 92(1), 192-200 (2015)

[7] Costabile, F.A., Gualtieri, M.I., Napoli, A.: Relationship between interpolation and differential equations: a class of collocation methods. Dynamical SystemsAnalytical and Computational Techniques, pp. 169-189. InTech (2017)

[8] Costabile, F.A.: Modern Umbral Calculus: An Elementary Introduction with Applications to Linear Interpolation and Operator Approximation Theory, vol. 72. Walter de Gruyter GmbH \& Co KG (2019)

[9] Costabile, F.A., Gualtieri, M.I., Napoli, A.: Lidstone-based collocation splines for odd-order bvps. Math. Comput. Simul. 186, 124-135 (2020) 
[10] Costabile, F.A., Gualtieri, M.I., Napoli, A.: Lidstone-Euler Interpolation and Related High Even Order Boundary Value Problem. Calcolo (2020) (submitted)

[11] Costabile, F.A., Gualtieri, M.I., Napoli, A.: Odd and even Lidstone-type polynomial sequences. Part 2: applications. Calcolo 57(1), 6 (2020)

[12] Costabile, F.A., Gualtieri, M.I., Napoli, A., Altomare, M.: Odd and even Lidstone-type polynomial sequences. Part 1: basic topics. Adv. Differ. Equ. 2018(1), 299 (2018)

[13] Costabile, F.A., Napoli, A.: Collocation for high-order differential equations with Lidstone boundary conditions. J. Appl. Math. 1-20, 2012 (2012)

[14] Costabile, F.A., Napoli, A.: Collocation for high order differential equations with two-points Hermite boundary conditions. Appl. Numer. Math. 87, 157$167(2015)$

[15] Costabile, F.A., Napoli, A.: A multipoint Birkhoff type boundary value problem. J. Numer. Math. 23(1), 1-11 (2015)

[16] Costabile, F.A., Napoli, A.: A class of Birkhoff-Lagrange-collocation methods for high order boundary value problems. Appl. Numer. Math. 116, 129-140 (2017)

[17] Davies, A.R., Karageorghis, A., Phillips, T.N.: Spectral Galerkin methods for the primary two-point boundary value problem in modelling viscoelastic flows. Int. J. Numer. Methods Eng. 26(3), 647-662 (1988)

[18] Davis, P.J.: Interpolation and Approximation (Courier Corporation, 1975)

[19] Henderson, J.: Multiple solutions for $2 \mathrm{~m}$-th order Sturm-Lioville boundary value problems on a measure chain. J. Differ. Equ. Appl. 6(4), 417-429 (2000)

[20] Hossain, B., Islam, S.: A novel numerical approach for odd higher order boundary value problems. Math. Theory Model. 4(5), 1-11 (2014)

[21] Jordan, C.: Calculus of Finite Differences, vol. 33 (American Mathematical Society, 1965)

[22] Liu, Y.: Solutions of two-point boundary value problems for even-order differential equations. J. Math. Anal. Appl. 323(1), 721-740 (2006)

[23] Lyshevski, S.E., Dunipace, K.R.: Identification and tracking control of aircraft from real-time perspectives. In: Proceedings of the 1997 IEEE International Conference on Control Applications. IEEE, pp. 499-504 (1997)

[24] Pethe, S.P., Sharma, A.: Modified Abel expansion and a subclass of completely convex functions. SIAM J. Math. Anal. 3(3), 546-558 (1972)

[25] Poritsky, H.: On certain polynomial and other approximations to analytic functions. Trans. Am. Math. Soc. 34(2), 274-331 (1932)

[26] Richards, G., Sarma, P.R.R.: Reduced order models for induction motors with two rotor circuits. IEEE Trans. Energy Convers. 9(4), 673-678 (1994)

[27] Schoenberg, I.J.: On certain two-point expansions of integral functions of exponential type. Bull. Am. Math. Soc. 42(4), 284-288 (1936)

[28] Schoenberg, I.J.: On Hermite-Birkhoff interpolation. J. Math. Anal. Appl. 16(3), 538-543 (1966)

[29] Siddiqi, S.S., Akram, G.: Solution of fifth order boundary value problems using nonpolynomial spline technique. Appl. Math. Comput. 175(2), 1574-1581 (2006)

[30] Siddiqi, S.S., Akram, G.: Sextic spline solutions of fifth order boundary value problems. Appl. Math. Lett. 20(5), 591-597 (2007) 
[31] Siddiqi, S.S., Akram, G., Iftikhar, M.: Solution of seventh order boundary value problems by variational iteration technique. Appl. Math. Sci. 6(94), 4663-4672 (2012)

[32] Stoer, J., Bulirsch, R.: Introduction to Numerical Analysis, vol. 12 (Springer Science \& Business Media, 2013)

[33] Viswanadham, K.N.S.K., Reddy, S.M.: Numerical solution of ninth order boundary value problems by Petrov-Galerkin method with quintic B-splines as basis functions and septic B-splines as weight functions. Procedia Eng. 127, 1227-1234 (2015)

[34] Wazwaz, A.M.: Approximate solutions to boundary value problems of higher order by the modified decomposition method. Comput. Math. Appl. 40(6-7), 679-691 (2000)

[35] Wazwaz, A.M.: The numerical solution of fifth-order boundary value problems by the decomposition method. J. Comput. Appl. Math. 136(1-2), 259-270 (2001)

[36] Whittaker, J.M.: On Lidstone's series and two-point expansions of analytic functions. Proc. Lond. Math. Soc. 2(1), 451-469 (1934)

Francesco Aldo Costabile, Maria Italia Gualtieri and Anna Napoli

Department of Mathematics and Computer Science

University of Calabria

87036 Rende CS

Italy

e-mail: anna.napoli@unical.it

Francesco Aldo Costabile

e-mail: francesco.costabile@unical.it

Maria Italia Gualtieri

e-mail: mariaitalia.gualtieri@unical.it

Received: November 1, 2020.

Revised: May 19, 2021.

Accepted: June 9, 2021. 